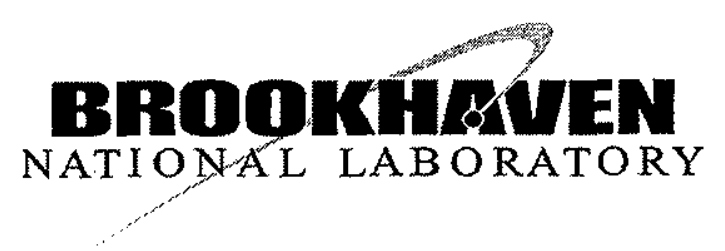

BNL-79508-2007-CP

\title{
Polarized proton acceleration in AGS and RHIC
}

\section{Thomas Roser}

Presented at the $12^{\text {th }}$ International Workshop on Polarized Sources, Targets, and Polarimetry

Brookhaven National Laboratory, Upton, NY

September $10-14,2007$

\author{
Collider-Accelerator Department \\ Brookhaven National Laboratory \\ P.O. Box 5000 \\ Upton, NY 11973-5000 \\ www.bnl.gov
}

\begin{abstract}
Notice: This manuscript has been authored by employees of Brookhaven Science Associates, LLC under Contract No. DE-AC02-98CH10886 with the U.S. Department of Energy. The publisher by accepting the manuscript for publication acknowledges that the United States Government retains a non-exclusive, paid-up, irrevocable, world-wide license to publish or reproduce the published form of this manuscript, or allow others to do so, for United States Government purposes.
\end{abstract}




\section{DISCLAIMER}

This report was prepared as an account of work sponsored by an agency of the United States Government. Neither the United States Government nor any agency thereof, nor any of their employees, nor any of their contractors, subcontractors, or their employees, makes any warranty, express or implied, or assumes any legal liability or responsibility for the accuracy, completeness, or any third party's use or the results of such use of any information, apparatus, product, or process disclosed, or represents that its use would not infringe privately owned rights. Reference herein to any specific commercial product, process, or service by trade name, trademark, manufacturer, or otherwise, does not necessarily constitute or imply its endorsement, recommendation, or favoring by the United States Government or any agency thereof or its contractors or subcontractors. The views and opinions of authors expressed herein do not necessarily state or reflect those of the United States Government or any agency thereof. 


\title{
Polarized Proton Acceleration in AGS and RHIC ${ }^{1}$
}

\author{
Thomas Roser \\ Brookhaven National Laboratory, Upton, New York 11793-5000, USA
}

\begin{abstract}
As the first hadron accelerator and collider consisting of two independent superconducting rings RHIC has operated with a wide range of beam energies and particle species including polarized proton beams. The acceleration of polarized beams in both the injector and the collider rings is complicated by numerous depolarizing spin resonances. Partial and full Siberian snakes have made it possible to overcome the depolarization and beam polarizations of up to $65 \%$ have been reached at $100 \mathrm{GeV}$ in RHIC.
\end{abstract}

Keywords: Polarized beams

PACS: 29.27.Hj, 29.20.dk

\section{THE RHIC FACILITY}

With its two independent rings RHIC is a highly flexible collider of hadron beams ranging from colliding intense beams of polarized protons to colliding fully stripped gold ions. The collision of $100 \mathrm{GeV} /$ nucleon gold ions probes the conditions of the early universe by producing extreme conditions where quarks and gluons are predicted to form a new state of matter. Several runs of high luminosity gold-gold collisions as well as comparison runs using proton, deuteron and copper beams have demonstrated that indeed a new state of matter with extreme density is formed in the RHIC gold-gold collisions.

The RHIC polarized proton collider has opened up the completely unique physics opportunities of studying spin effects in hadronic reactions at high-luminosity highenergy proton-proton collisions. It allows the study of the spin structure of the proton, in particular the degree of polarization of the gluons and anti-quarks, and also verification of the many well-documented expectations of spin effects in perturbative QCD and parity violation in $\mathrm{W}$ and $\mathrm{Z}$ production. The RHIC center-of-mass energy range of 200 to $500 \mathrm{GeV}$ is ideal in the sense that it is high enough for perturbative QCD to be applicable and low enough so that the typical momentum fraction of the valence quarks is about 0.1 or larger. This guarantees significant levels of parton polarization.

During its first seven years of operation RHIC has already exceeded the design parameters for gold-gold collisions, has successfully operated in an asymmetric mode of colliding deuteron on gold with both beams at the same energy per nucleon but, of course, different rigidities, and very successfully completed an additional comparison run of

\footnotetext{
1 Work performed under the auspices of the United States Department of Energy, and with support of RIKEN (Japan) and Renaissance Technologies Corp. (USA)
} 


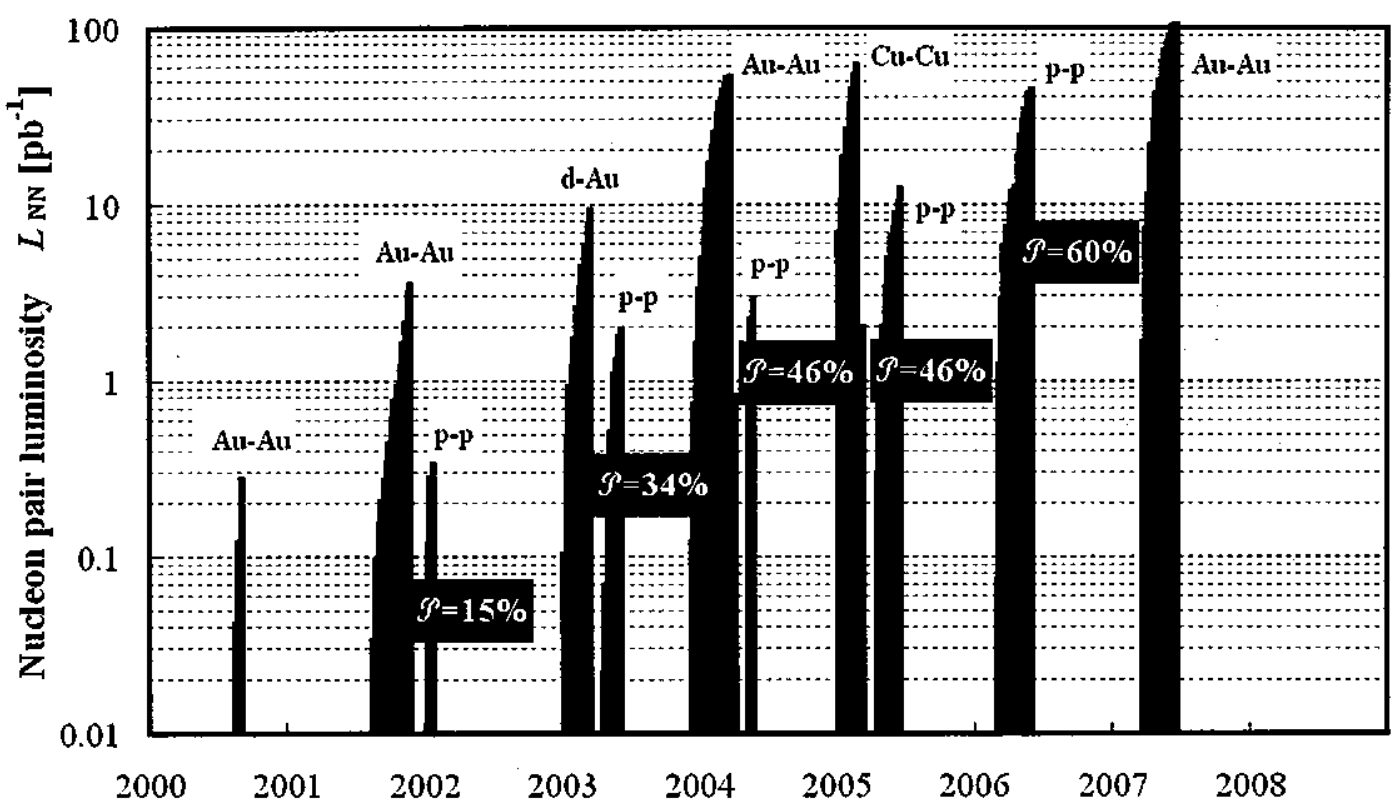

FIGURE 1. Integrated nucleon-pair luminosity for all the RHIC running modes since start of operation.

colliding copper beams with record luminosities. In addition, four very successful commissioning and running periods with polarized protons demonstrated the performance of RHIC as a high luminosity polarized collider. For the main part of all these runs RHIC was operating with beam energies of $100 \mathrm{GeV} /$ nucleon - the gold beam design energy. Additional running at lower beam energy was also accomplished during these same running periods again demonstrating the high flexibility of RHIC. Fig. 1 shows, in semi-logarithmic scale, the achieved integrated nucleon-pair luminosities for the many modes of operation of RHIC since its start of operation in 2000. Using nucleon-pair luminosity allows the comparison of the different modes properly reflecting the relative statistical relevance of the data samples and also the degree of difficulty in achieving high luminosity.

\section{SPIN DYNAMICS, RESONANCES AND SIBERIAN SNAKES}

Accelerating polarized beams requires an understanding of both the orbital motion and spin motion. Whereas the effect of the spin on the orbit is negligible the effect of the orbit on the spin is usually very strong. The evolution of the spin direction of a beam of polarized protons in external magnetic fields such as exist in a circular accelerator is governed by the Thomas-BMT equation [1],

$$
\frac{d \vec{P}}{d t}=-\left(\frac{e}{\gamma m}\right)\left[G \gamma \overrightarrow{B_{\perp}}+(1+G) \overrightarrow{B_{\|}}\right] \times \vec{P}
$$

where the polarization vector $P$ is expressed in the frame that moves with the particle. This simple precession equation is very similar to the Lorentz force equation which 


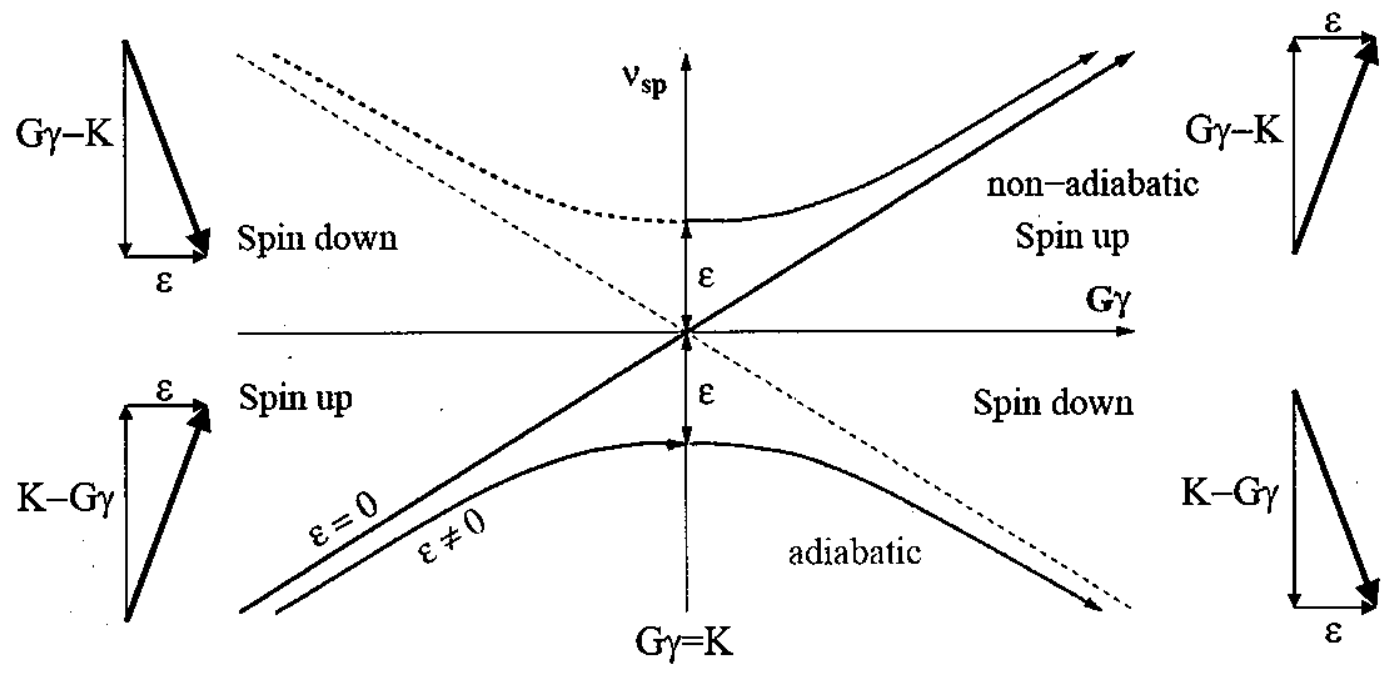

FIGURE 2. The evolution of the spin tune during the crossing of a resonance at $G /$ gamma $=K$ with strength $\varepsilon$.

governs the evolution of the orbital motion in an external magnetic field:

$$
\frac{d \vec{v}}{d t}=-\left(\frac{e}{\gamma m}\right)\left[\overrightarrow{B_{\perp}}\right] \times \vec{v} .
$$

From comparing these two equations it can readily be seen that, in a pure vertical field, the spin rotates $G \gamma$ times faster than the orbital motion. Here $G=1.7928$ is the anomalous magnetic moment of the proton and $\gamma=E / m$. In this case the factor $G \gamma$ then gives the number of full spin precessions for every full revolution, a number which is also called the spin tune $v_{s p}$. At top RHIC energies this number reaches about 400 . The Thomas-BMT equation also shows that at low energies $(\gamma \approx 1)$ longitudinal fields $\overrightarrow{B_{\|}}$ can be quite effective in manipulating the spin motion, but at high energies transverse fields $\overrightarrow{B_{\perp}}$ need to be used to have any effect beyond the always present vertical holding field.

The acceleration of polarized beams in circular accelerators is complicated by the presence of numerous depolarizing spin resonances. During acceleration, a spin resonance is crossed whenever the spin precession frequency equals the frequency with which spin-perturbing magnetic fields are encountered. There are two main types of spin resonances corresponding to the possible sources of such fields: imperfection resonances, which are driven by magnet errors and misalignments, and intrinsic resonances, driven by the focusing fields.

The resonance conditions are usually expressed in terms of the spin tune $v_{s p}$. For an ideal planar accelerator, where orbiting particles experience only the vertical guide field, the spin tune is equal to $G \gamma$, as stated earlier. The resonance condition for imperfection depolarizing resonances arise when $v_{s p}=G \gamma=n$, where $n$ is an integer. Imperfection resonances are therefore separated by only $523 \mathrm{MeV}$ energy steps. The condition for 
intrinsic resonances is $v_{s p}=G \gamma=k P \pm v_{y}$, where $k$ is an integer, $v_{y}$ is the vertical betatron tune and $P$ is the superperiodicity. For example at the AGS, $P=12$ and $v_{y} \approx 8.8$.

Close to a spin resonance the spin tune deviates away from its value of $G \gamma$ of the ideal flat machine. For a resonance with strength $\varepsilon$, which is the total spin rotation due to the resonance driving fields, the new spin tune is given by the equation

$$
\cos \left(\pi v_{s p}\right)=\cos (\pi G \gamma) \cos (\pi \varepsilon)
$$

Fig. 2 shows the solutions of this equation with and without a resonance. A similar calculation can be done for the effective precession direction or, as it is often called, the stable spin direction. The stable spin direction describes those polarization components that are repeated every turn. Note that both the stable spin direction and the spin tune are completely determined by the magnetic structure of the accelerator and the beam energy. The magnitude and sign of the beam polarization, however, depends on the beam polarization at injection and the history of the acceleration process.

The spin tune and stable spin direction calculations apply only to a time-independent static situation or if parameters are changed adiabatically. Far from the resonance the stable spin direction coincides with the main vertical magnetic field. Close to the resonance at $G /$ gamma $=K$, the stable spin direction is perturbed away from the vertical direction by the resonance driving fields. When a polarized beam is accelerated through an isolated resonance at arbitrary speed, the final polarization can be calculated analytically [2] and is given by

$$
P_{f} / P_{i}=2 e^{-\frac{\pi|\varepsilon|^{2}}{2 \alpha}}-1
$$

where $P_{i}$ and $P_{f}$ are the polarizations before and after the resonance crossing, respectively, and $\alpha$ is the change of the spin tune per radian of the orbit angle. When the beam is slowly $\left(\alpha \ll|\varepsilon|^{2}\right)$ accelerated through the resonance, the spin vector will adiabatically follow the stable spin direction resulting in spin flip as is indicated in Fig. 2. However, for a faster acceleration rate partial depolarization or partial spin flip will occur.

Traditionally, the intrinsic resonances are overcome by using a betatron tune jump, which effectively makes $\alpha$ large, and the imperfection resonances are overcome with the harmonic corrections of the vertical orbit to reduce the resonance strength $\varepsilon$ [3]. Both of these methods aim at making the resonance crossing non-adiabatic. They require very accurate adjustments at every resonance crossing, which can become very difficult and time consuming.

New techniques to cross both imperfection and intrinsic resonances adiabatically have been developed. The correction dipoles used to correct the imperfection resonance strength to zero were replaced by a localized spin rotator or 'partial Siberian snake' which makes all the imperfection resonance strengths large and causes complete adiabatic spin flip at every imperfection resonance [4]. The tune jump quadrupoles were initially replaced by a single rf dipole magnet, which increased the strength of the intrinsic resonances by driving large coherent betatron oscillations[5].

More recently two strong partial Siberian snakes were installed in the AGS that can overcome both imperfection and intrinsic resonances. With strong enough partial snakes a gap between the spin tune and an integer opens up that becomes large enough to place the fractional part of the betatron tune and therefore the intrinsic resonance inside this 


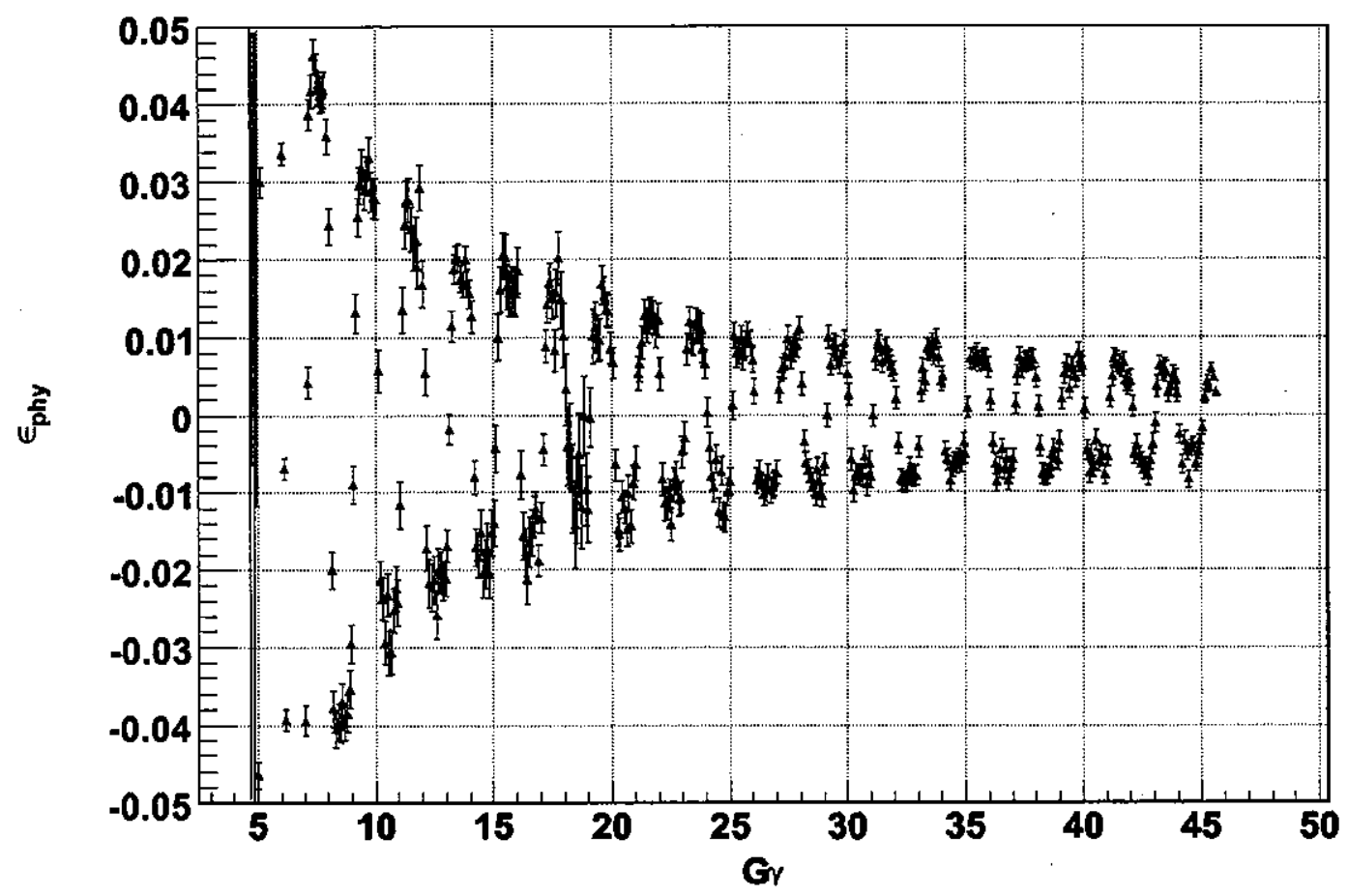

FIGURE 3. Left-right asymmetry measured by the AGS polarimeter during the the acceleration cycle. Note that the asymmetry flips sign at every interger value of $\mathrm{G} \gamma$. The drop of the magnitude of the asymmetry during acceleration is mainly due to the decreasing analyzing power of the analyzing reaction of small-angle proton-carbon scattering.

gap. Fig. 3 shows the measured asymmetry during acceleration in the AGS showing adiabatic spin flip at every integer of $\mathrm{G} \gamma$.

At higher energies a 'full Siberian snake' [6], which is a $180^{\circ}$ spin rotator of the spin about a horizontal axis, will keep the stable spin direction unperturbed at all times as long as the spin rotation from the Siberian snake is much larger than the spin rotation due to the resonance driving fields. Therefore the beam polarization is preserved during acceleration. An alternative way to describe the effect of the Siberian snake comes from the observation that the spin tune with the snake is a half-integer and energy independent. Therefore, neither imperfection nor intrinsic resonance conditions can ever be met as long as the betatron tune is different from a half-integer.

\section{POLARIZED PROTON COLLISIONS}

Fig. 4 shows the lay-out of the Brookhaven accelerator complex highlighting the components required for polarized beam acceleration. The new 'Optically Pumped Polarized Ion Source'[7] is producing $10^{12}$ polarized protons per pulse. A single source pulse is captured into a single bunch, which is ample beam intensity to reach the nominal RHIC bunch intensity of $2 \times 10^{11}$ polarized protons. 


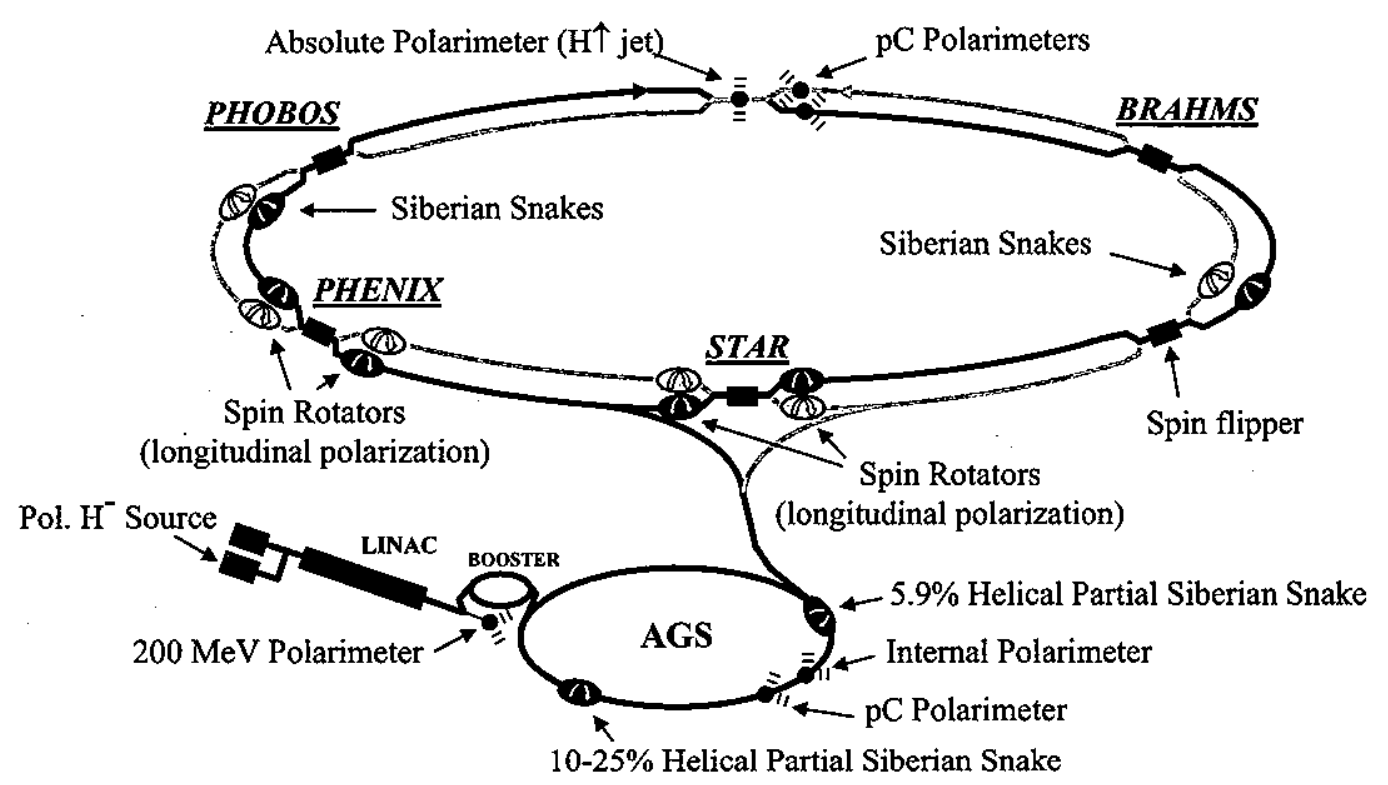

FIGURE 4. The RHIC accelerator complex with the elements required for the acceleration and collision of polarized protons highlighted.

In the AGS two partial Siberian snakes are installed. One of them is an iron-based helical dipole[8] that rotates the spin by $11^{\circ}$. A view down the magnet gap is shown in Fig. 5. The other is a superconducting helical dipole that can reach a 3 Tesla field and a spin rotation of up to $45^{\circ}$. Both helical dipoles have the same design with a variable pitch along the length of the magnet to minimize orbit excursions and also to fit into the $3 \mathrm{~m}$ available straight sections in the AGS. With the two partial snakes strategically placed with one third of the AGS ring between them all vertical spin resonances can be avoided up to the required RHIC transfer energy of about $25 \mathrm{GeV}$ as long as the vertical betatron tune is placed at 8.98 , very close to an integer[9]. This was achieved reliably over the whole acceleration cycle. With a $80 \%$ polarization from the source $65 \%$ polarization was reached at AGS extraction. The remaining polarization loss in the AGS might come from weak spin resonances driven by the horizontal motion of the beam. They could be overcome by moving also the horizontal betatron tune close to an integer.

The full Siberian snakes (two for each ring) and the spin rotators (four for each collider experiment) for RHIC each consist of four $2.4 \mathrm{~m}$ long, $4 \mathrm{~T}$ helical dipole magnet modules each having a full $360^{\circ}$ helical twist. The $9 \mathrm{~cm}$ diameter bore of the helical magnets can accommodate $3 \mathrm{~cm}$ orbit excursions at injection. Fig. 6 shows the orbit and spin trajectory through a RHIC snake. The super-conducting helical dipoles for both the RHIC snakes and spin-rotators and the superconducting AGS partial snake were constructed at BNL using thin cable placed into helical grooves that have been milled into a thick-walled aluminum cylinder[10].

In addition to maintaining polarization, the accurate measurement of the beam polarization is of great importance. Very small angle elastic scattering in the Coulomb- 


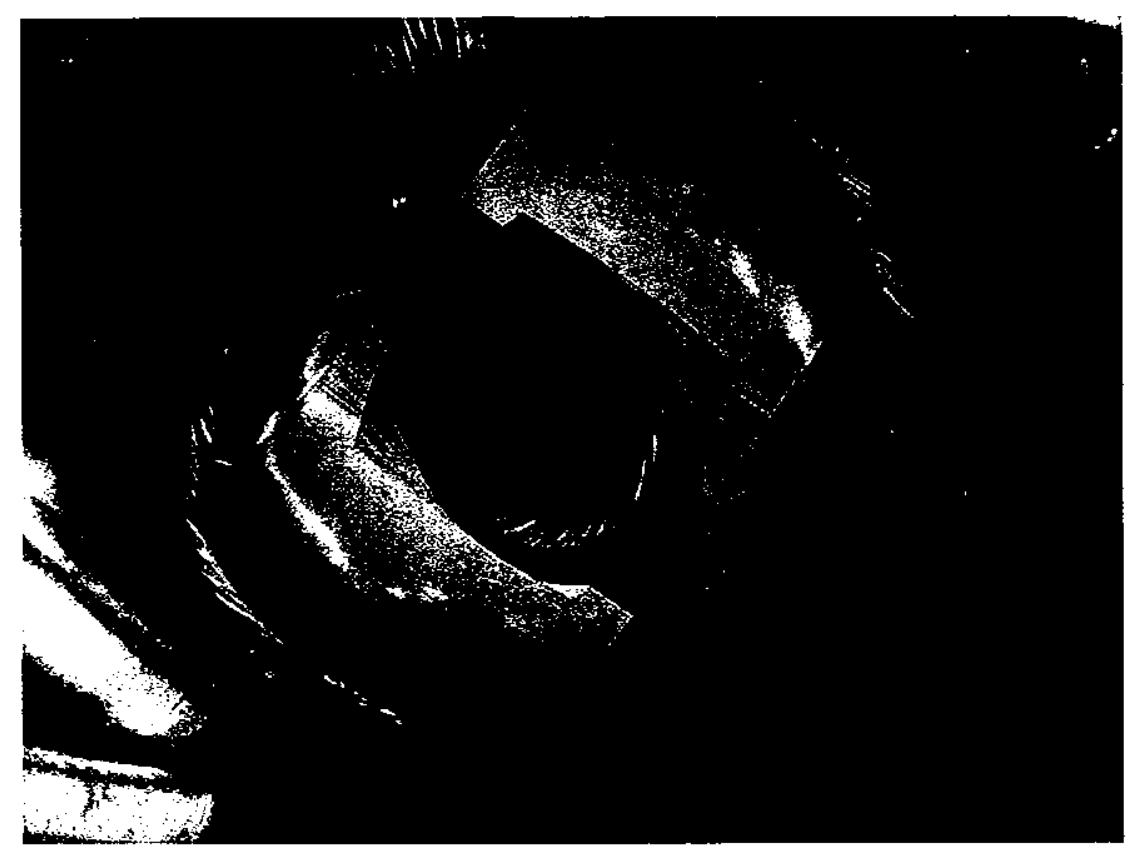

FIGURE 5. View down the magnet gap of the warm, iron-based helical partial Siberian snake of the AGS.

Nuclear interference region offers the possibility for an analyzing reaction with a high figure-of-merit, which is not expected to be strongly energy dependent[11]. For polarized beam commissioning in the AGS and RHIC an ultra-thin carbon ribbon is used as an internal target, and the recoil carbon nuclei are detected to measure both vertical and radial polarization components. The detection of the recoil carbon with silicon detectors using both energy and time-of-flight information shows excellent particle identification.

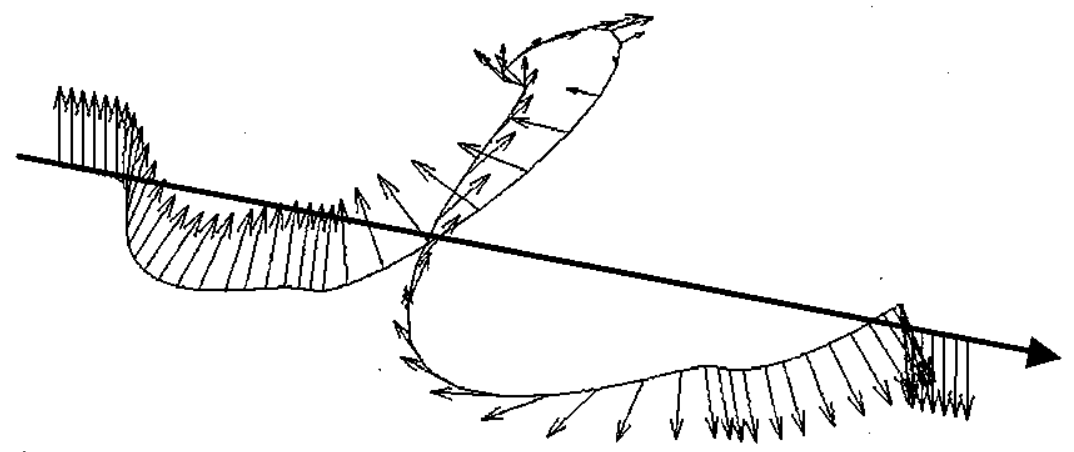

FIGURE 6. Orbit and spin tracking through the four helical magnets of a Siberian Snake at $\gamma=25$. The spin tracking shows the reversal of the vertical polarization. 


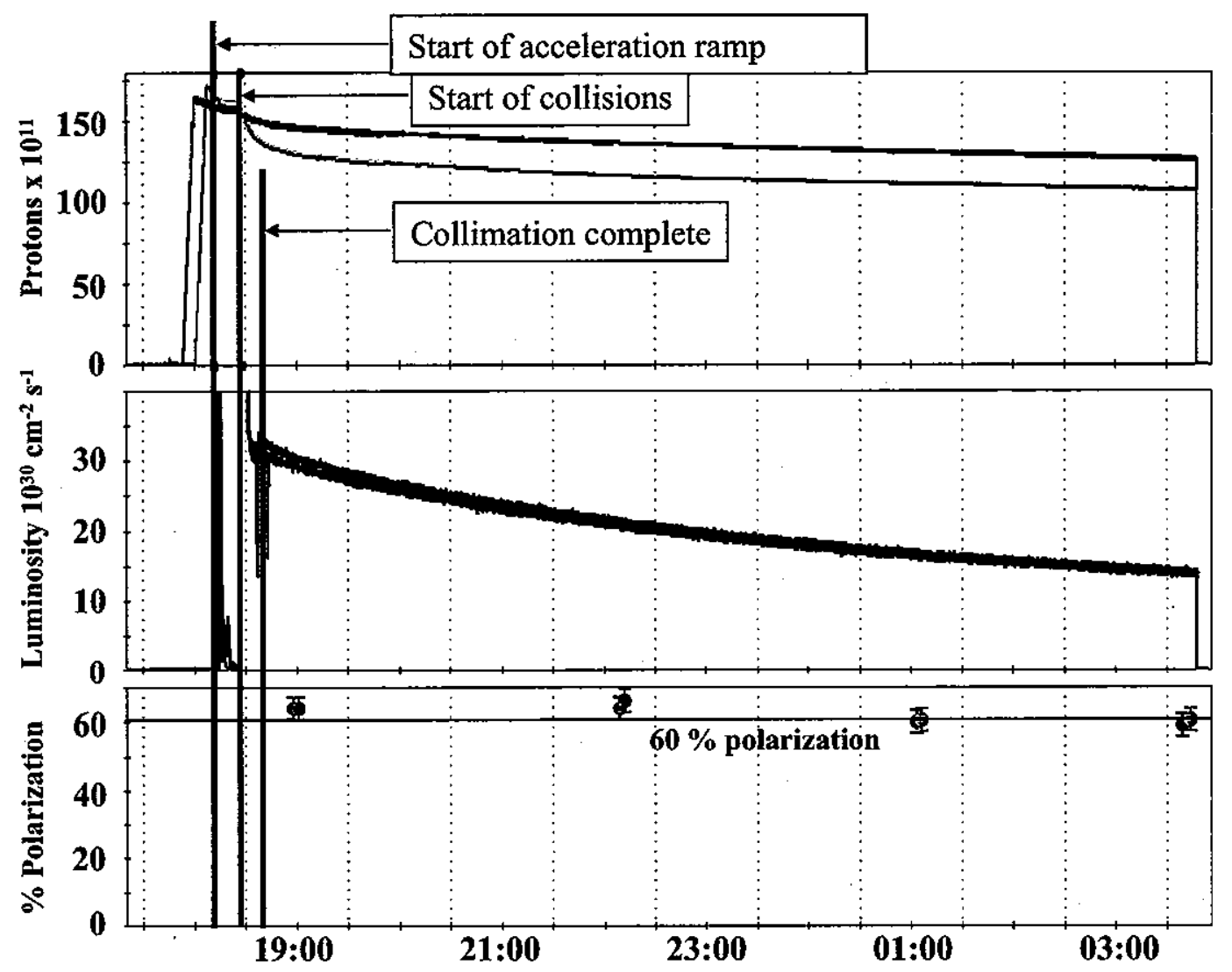

FIGURE 7. Circulating beam in the blue and yellow ring, luminosity at PHENIX (black) and STAR (red), as well as the measured circulating beam polarization in the blue and yellow RHIC ring (blue(dark) and yellow(light) lines and symbols, respectively) for one typical store.

It was demonstrated that this polarimeter can be used to monitor polarization of high energy proton beams in an almost non-destructive manner and that the carbon fiber target could be scanned through the circulating beam to measure polarization profiles. A polarized atomic hydrogen jet was also installed as an internal target for small angle proton-proton scattering, which allows the absolute calibration of the beam polarization to better than $5 \%$.

Fig. 7 shows circulating beam current, luminosity and measured circulating beam polarization of a typical store during the 2006 run[12]. A peak luminosity of about about $35 \times 10^{30} \mathrm{~cm}^{-2} \mathrm{~s}^{-1}$ was reached. The beam polarization of up to $65 \%$ was calibrated at $100 \mathrm{GeV}$ with the absolute polarimeter mentioned above. To preserve beam polarization in RHIC during acceleration and storage the vertical betatron tune had to be controlled to better than 0.005 and the orbit had to be corrected to better than $1 \mathrm{~mm}$ rms to avoid depolarizing "snake" resonances[13].

More than 20 years after Y. Derbenev and A. Kodratenko[6] made their proposal to use local spin rotators to stabilize polarized beams in high energy rings, it has now been demonstrated that their concept is working flawlessly even in the presence of strong spin 

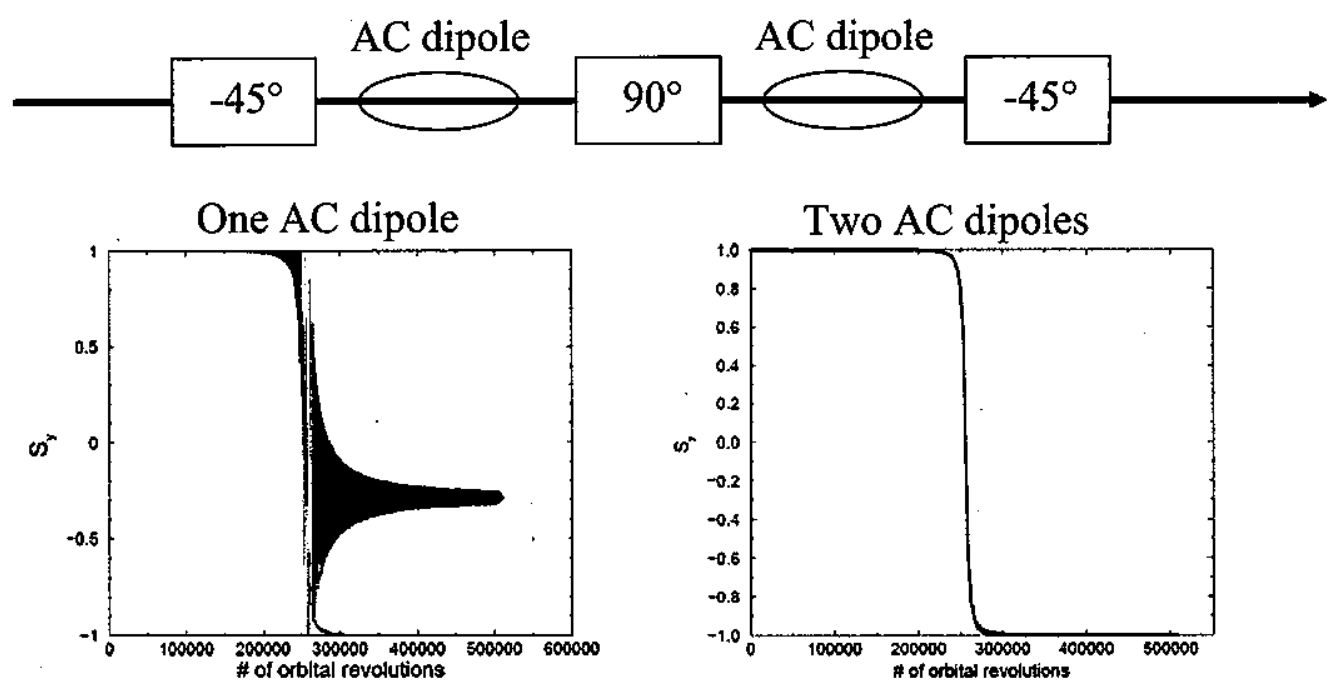

FIGURE 8. Magnet arrangement for RHIC spin flipper and rtacking results for a single ac dipole and two ac dipoles.

resonances at high energy.

A first successful test of polarization survival during acceleration to $250 \mathrm{GeV}$ crossing three very strong spin resonances was performed[14]. A polarization of $45 \%$ was measured at $250 \mathrm{GeV}$ using the pC polarimeter calibrated at $100 \mathrm{GeV}$. It is not expected that the calibration would change significantly between these two energies, but a more accurate polarization will have to await a calibration measurement with the polarized gas jet performed at $250 \mathrm{GeV}$. Nevertheless, this preliminary result bodes well for a successful operation of RHIC with polarized $250 \mathrm{GeV}$ proton beams producing collisions at $\sqrt{s}=500 \mathrm{GeV}$ with a planned luminosity of up to $1.5 \times 10^{32} \mathrm{~cm}^{-2} \mathrm{~s}^{-1}$.

\section{SPIN MANIPULATION AND FLIPPING}

Artificially driven spin resonances can be used to control the polarization of the stored proton beam. Typically a horizontal magnetic dipole field that is modulated at or close to the spin tune frequency is used. These devices are called either $\mathrm{rf}$ or ac dipoles depending on the frequency of modulation. As described above an rf dipole was used in the AGS to overcome intrinsic resonances. By ramping the drive frequency through the resonance condition a full spin flip can be achieved [15]. For RHIC with a spin tune of 0.5 the two resonances that a simple rf dipole produces interfere during the resonance crossing and prevent a full spin flip. A new device that consists of two ac dipoles with a spin rotator in between can avoid this problem by producing only a single resonance by properly adjusting the relative amplitude and phase of the ac dipole excitations[16]. Fig. 8 shows the magnet arrangement and the tracking results with a single ac dipole and two ac dipoles. Full spin flip can only be achieved with two ac dipoles.

This device can also be used to measure the spin tune. By adiabatically exciting the 
spin flipper at a drive tune close to the spin tune the beam polarization will be tilted away from the otherwise stable vertical direction. If the strength of the spin flipper is larger than the spin tune spread the whole beam polarization will tilt in the same way and can be measured with the polarimeter. The ratio of the vertical and horizontal polarization component is then proportional to the difference between the drive tune and the spin tune.

\section{ACKNOWLEDGMENT}

The highly successful operation of the RHIC polarized collider was made possible by the excellent and dedicated RHIC design, construction, commissioning, and operations teams.

\section{REFERENCES}

1. L.H. Thomas, Phil. Mag. 3, 1 (1927); V. Bargmann, L. Michel, V.L. Telegdi, Phys. Rev. Lett. 2, 435 (1959).

2. M. Froissart and R. Stora, Nucl. Instr. Meth., 1, 297 (1960).

3. T. Khoe et al., Part. Accel. 6, 213 (1975); J.L. Laclare et al., J. Phys. (Paris), Colloq. 46, C2-499 (1985); H. Sato et al., Nucl. Inst. Meth., Phys. Res. Sec A272, 617 (1988); F.Z. Khiari, et al., Phys. Rev. D39, 45 (1989).

4. T. Roser, AIP Conf. Proc. No. 187, ed. K.J. Heller p.1442 (AIP, New York, 1988).

5. M. Bai et al., Phys. Rev. Lett. 80, 4673 (1998)

6. Ya.S. Derbenev et al., Part. Accel. 8, 115 (1978).

7. J. Alessi et al., "Polarized H- Source Performance during the 2003 RHIC Run", Proc. of PAC03, Portland, Oregon, p. 3282.

8. J. Takano et al., "Optimization of AGS Polarized Proton Operation with the Warm Helical Snake", Proc. of PAC05, Knoxville, Tennessee, p. 1003.

9. H. Huang et al., Phys. Rev. Lett. 99, 146802 (2007)

10. E. Willen et al., "Superconducting Helical Snake Magnet for the AGS", Proc. of PAC05, Knoxville, Tennessee, p. 2935.

11. J. Tojo et al., Phys. Rev. Lett. 89, 052302 (2002).

12. V. Ptitsyn et al., "RHIC Performance as Polarized Proton Collider in Run-6", Proc. of EPAC 2006, Edinburgh, Scotland, p. 592.

13. M. Bai et al., Phys. Rev. Lett. 96, 174801 (2006); M. Bai et al., "Observations of Snake Resonance in RHIC", Proceedings of PAC05, Knoxville, Tennessee, p. 2839.

14. M. Bai et al., Phys. Rev. Lett. 96, 174801 (2006); M. Bai et al., "Accelerating Polarized Protons to High Energy", Proc. of SPIN2006, to be published.

15. D.D. Caussyn et al., Phys. Rev. Lett. 73, 2857 (1994); B. B. Blinov et al., Phys. Rev. Lett. 81, 2906 (1998); B. B. Blinov et al., Phys. Rev. Lett. 88, 014801 (2002); V. S. Morozov et al., Phys. Rev. ST Accel. Beams 7, 024002 (2004).

16. M. Bai et al., "RHIC Spin Flipper", Proceedings of PAC07, Albuquerque, New Mexico, p.1847. 